\title{
Modèle numérique de clapage - phase de chute
}

\author{
Isabelle Farout-Fréson ${ }^{1}$, Philippe Sergent ${ }^{2}$, Emmanuel Lefrançois ${ }^{1}$, \\ Gouri Dhatt ${ }^{1}$ \\ ${ }^{1}$ UTC LHN Roberval 60200 Compiègne \\ ${ }^{2}$ CETMEF 2, boulevard Gambetta 60321 Compiègne cedex
}

\begin{abstract}
Résumé. Nous nous intéressons ici aux mélanges fluide incompressible/ particules solides fluidisées. Le modèle proposé est basé sur l'écriture moyennée des équations hydrodynamiques bi phasiques associée à une équation de transport de sédiments munie d'une vitesse numérique de chute $\mathrm{w}_{\mathrm{sf}}$ spécifiquement adaptée au cas des rejets de dragage par clapage. La stratégie de résolution entre l'hydrodynamique et le transport est réalisée en considérant une variation de la densité du mélange de façon explicite afin d'obtenir un modèle couplé de manière séquentielle. Calibré sur la phase de chute de trois campagnes expérimentales de clapage en canal, le modèle donne de très bons résultats sur près de vingt essais de rejets sableux, sablo vaseux, vaseux avec et sans courant ambiant.
\end{abstract}

\begin{abstract}
We are interested here in the mixture composed of incompressible fluid and a certain mass of fluidised solid. The proposed model is based on the averaged form of the hydrodynamic biphasic equations, associated with a sediment transport equation with a specific numerical settling velocity $\mathrm{w}_{\mathrm{sf}}$ adapted for the dumped dredged material case. Both models (hydrodynamics and transport) are coupled considering the variation of density with a forward scheme. Calibrated on the convective descent on three experimental campaigns in canal of dumping of dredged materials, the model gives a very good agreement of convective descent with almost twenty experiments for materials $100 \%$ sand, $100 \%$ silt or mixture sand/silt without or with a horizontally ambient current.
\end{abstract}

Mots clés. Rejets de dragage, mélange fluide sédiments, densité variable, vitesse de chute.

\section{Introduction}

Les ports maritimes et voies navigables sont régulièrement soumis à des envasements qui rendent la navigation difficile voire dangereuse. Afin de faciliter les trafics maritimes et fluviaux, chaque port procède à des dragages d'entretien qui représentent par an et en France un volume de rejets de plus de 50 millions de mètres cubes. Ces rejets sont composés essentiellement de sables, de boues, de vases mais aussi de polluants d'origines variées. Du point de vue réglementaire, les conditions d'immersion des rejets de dragage sont fixées au niveau international par la convention d'Oslo. La prise en compte de ces dispositions en 1996 amène les autorités françaises à missionner un groupe de travail sur les

DOI: 10.5150/jngcgc.2006.018-F $\quad$ (disponible en ligne - http://www.paralia.fr - available online) 
opérations de dragage et son impact sur l'environnement pour faire le point sur les connaissances scientifiques en matière d'impact des opérations de dragage et d'immersion, établir la demande en besoin de recherche et proposer un programme de recherche pour combler les lacunes identifiées. De cette mission naît le projet «Liteau clapage» dont l'objectif est de recenser les outils de prédiction disponibles pour étudier l'impact des rejets de dragage sur le littoral. Des essais physiques ont en outre été réalisés de 1997 à 2001 au sein du LNHEEDF à Chatou (France) pour des rejets $100 \%$ sable (Burel et Villaret ${ }^{4}$, Villaret et al. $\left.{ }^{14}\right)$, sablo vaseux et $100 \%$ vase $\left(\right.$ Boutin $\left.^{2}\right)$. Une synthèse des modèles de rejet de dragage (Krishnappan ${ }^{9}$, STFATE $^{3}$, Drapeau et al. ${ }^{6}$ entre autres) montre un déficit de modèle pour les sites d'immersion de faible profondeur (inférieure à 100 mètres) avec courant ambiant qui représentent la quasi totalité des sites d'immersion français. Le travail, présenté ici, tente de palier cette lacune en portant donc une attention toute particulière aux rejets de dragage sur les sites de clapage français dont la profondeur n'excède pas soixante mètres et pour lesquels les courants de fond ou de surface peuvent être importants. L'analyse de Roland Boutin sur de tels rejets montre que les vitesses de chute observés en milieu naturel, sont très élevées et comprises entre 25 et $280 \mathrm{~cm} / \mathrm{s}$ et ne peuvent donc être modélisées par les lois classiques de vitesse de chute sédimentaire exprimées par bon nombre d'auteurs (Richardson et $\mathrm{Zaki}^{11}$, Tambo et Watanabe ${ }^{12}$, Migniot ${ }^{10}$, Van Leussen ${ }^{13}$ entre autres). Pour les cas qu'il présente, Gordon ${ }^{8}$ confirme également que la vitesse de chute du nuage d'une valeur moyenne de $40 \mathrm{~cm} / \mathrm{s}$, est nettement supérieure à la vitesse de chute des particules individuelles qui le composent, elle-même estimée à $0.5 \mathrm{~cm} / \mathrm{s}$. Le modèle numérique présenté ici est donc destiné à simuler le phénomène de chute du clapage pour des rejets $100 \%$ sable, sablo vaseux voire $100 \%$ vase avec ou sans courant ambiant.

L'approche la plus naturelle, l'approche bi phasique, permet de décrire les caractéristiques propres à chacune des phases du mélange en résolvant les équations de l'hydrodynamique sédimentaire pour l'eau et le sédiment. L'approche bi espèce utilisée ici, découle directement de l'approche bi phasique en reportant chacune des phases à sa part de masse au sein du mélange et permet de décrire non plus l'évolution moyenne de chaque phase mais l'évolution du mélange en tant que tel. Nous définissons ici brièvement le modèle numérique aux éléments finis bi espèce 2D (pour les détails voir Farout-Fréson ${ }^{7}$ ) pour 1'appliquer au cas du clapage avec la formulation d'une nouvelle vitesse de chute numérique spécifique dont les résultats sont comparés aux essais physiques expérimentaux faits au LNHE-EDF (Chatou France).

\section{Modèle bi espèce 2D de clapage}

Partant d'une approche bi phasique de l'écoulement du mélange avec une approche continue pour chaque phase représentée par les équations suivantes : 


$$
\begin{aligned}
& \text { Phase sédiment: } \begin{array}{l}
\frac{\partial \rho}{\partial t}+\operatorname{din}\left(\rho_{s}\right. \\
\text { Ph }+\operatorname{div}\left(\rho_{f} \vec{u}_{f}\right)=0 \\
\text { Phase fluide : } \quad \frac{\partial \rho_{f} \vec{u}_{f}}{\partial t}+\operatorname{div}\left(\rho_{f} \vec{u}_{f} \otimes \vec{u}_{f}-\bar{\sigma}_{f}\right)+\vec{F}_{f s}-\rho_{f} \vec{g}=\overrightarrow{0}
\end{array}
\end{aligned}
$$$$
\left\{\begin{array}{l}
\frac{\partial \rho_{s}}{\partial t}+\operatorname{div}\left(\rho_{s} \vec{u}_{s}\right)=0 \\
\frac{\partial \rho_{s} \vec{u}_{s}}{\partial t}+\operatorname{div}\left(\rho_{s} \vec{u}_{s} \otimes \vec{u}_{s}-\bar{\sigma}_{s}\right)+\vec{F}_{s f}-\rho_{s} \vec{g}=\overrightarrow{0}
\end{array}\right.
$$

Les termes $\vec{F}_{s f}$ et $\vec{F}_{f s}$ représentant les forces d'interaction entre la phase fluide et la phase solide et les tenseurs des contraintes sont évalués pour chaque composant selon la formulation d'Anderson et Jackson ${ }^{1}$. Les équations bi espèce s'obtiennent par une moyenne massique des équations bi phasiques, permettant d'obtenir un modèle mathématique comparable à un modèle mono phasique. Dans l'équation de conservation de la masse, la variation temporelle de la densité est négligée, et une méthode de pénalité sur le champ de pression (Dhatt et al. ${ }^{5}$ ) conduit à la relation suivante $\operatorname{div}\left(\rho \vec{u}^{(m)}\right)=\frac{p}{\lambda}$.

La vitesse du mélange est définie par pondération de la vitesse sédimentaire ${ }^{\vec{u}_{s}}$ et fluide $\vec{u}_{f}$ selon $\vec{u}^{(m)}=C \vec{u}_{s}+(1-C) \vec{u}_{f}$. En notant la vitesse en moyenne massique $\vec{u}^{(m)}=\vec{u}$, la conservation de quantité de mouvement s'écrit :

$\frac{\partial \rho \vec{u}}{\partial t}+\operatorname{div}(\rho \vec{u} \otimes \vec{u}+p \overline{\overline{1}}-2 \mu \overline{\bar{\varepsilon}})-\rho \vec{g}=\overrightarrow{0}$

Le transport de masse est donné par :

$\frac{\partial(\rho C)}{\partial t}+\operatorname{div}\left(\rho C\left(\left(\vec{u}^{(m)}+\vec{u}_{s f}\right)-k_{\text {diff }} \vec{\nabla} C\right)\right)=0$

Comme dans la théorie de la turbulence, la vitesse sédimentaire est ici décomposée en une vitesse moyenne $\vec{u}^{(m)}$ et une vitesse de fluctuation $\overrightarrow{\tilde{u}}_{s}$, qui gère tous les effets secondaires dus aux interactions entre les particules sédimentaires et le fluide. Les effets d'entraînement d'une phase par rapport à l'autre, qui se manifestent principalement par diffusion et convection sont modélisés par la seconde loi de Fick, quant aux autres, ils sont représentés par une vitesse d'entraînement supplémentaire $\vec{u}_{s f}$. Dans le cas du clapage cette vitesse

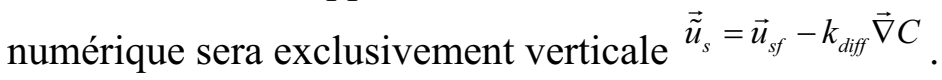

Le domaine de simulation est un rectangle d'un mètre sur quatre avec une concentration initiale homogène non nulle ${ }^{C_{0}}$ répartie sur dix sept centimètres de hauteur et cinq centimètres et demi de largeur. La position d'un rejet Y est repérée par sa position la plus basse avec un seuil de fraction massique fixé à un trentième de la fraction massique initiale et le rayon du rejet $\mathrm{R}$ correspond au cours de la chute à la largeur la plus importante du rejet avec un seuil de fraction massique fixé à un centième de la fraction massique initiale. Ces choix peuvent influer sensiblement sur les résultats. 


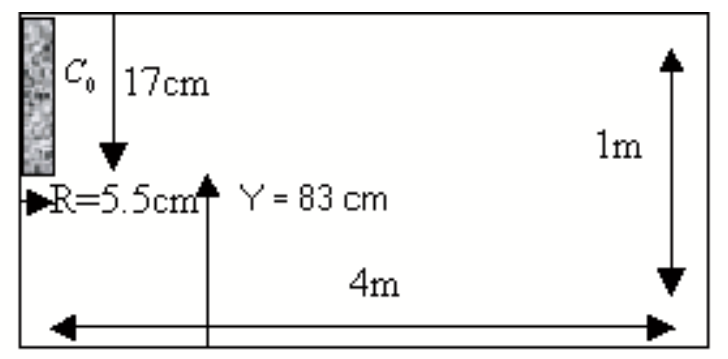

Figure 1. Domaine de simulation des essais sans courant ambiant $U_{a}=0 \mathrm{~m} / \mathrm{s}$ (Rejet symétrique).

Les simulations sont effectuées sur un demi domaine comportant un demi rejet pour les clapages sans courant ambiant Figure 1 (d'après les observations expérimentales). Pour les rejets avec courants ambiants, nous prenons l'intégralité du rejet; concentration homogène initiale non nulle $\mathrm{C}_{0}$ répartie sur un rectangle de dix sept centimètres de hauteur sur onze centimètres de largeur. La position initiale du rejet est fixée à $\mathrm{Y}=1-0,17=0,83 \mathrm{~m}$. Le rayon initial est de $\mathrm{R}=0,055 \mathrm{~m}$ pour les rejets sans courant et le diamètre initial $\mathrm{D}$ (largeur maximum effective de la masse sédimentaire en chute avec le même seuil de fraction massique que précédemment) est de $\mathrm{D}=0,11 \mathrm{~m}$ pour les rejets avec courant. Pour permettre une comparaison effective de nos résultats numériques avec ceux des essais (donnés toutes les $0,13 \mathrm{~s}$ ), nous avons choisi un pas de temps garantissant un maximum de données comparables soit $\Delta \mathrm{t}=0.1 \mathrm{~s}$. Le pas d'espace vertical $\Delta \mathrm{z}=0.0333 \mathrm{~m}$ est une valeur moyenne des différents écarts expérimentaux observés. Le pas d'espace horizontal, quant à lui $\Delta x=0.0392 \mathrm{~m}$ permet un temps de calcul relativement raisonnable pour un modèle écrit en Matlab $\subset$ : soit 1 min 45 s par pas de temps sur Pentium III cadencé à $1 \mathrm{Ghz}$.

$\rho_{f_{0}}=1000 \mathrm{~kg} / \mathrm{m}^{3} \quad \rho_{s_{0}}=2650 \mathrm{~kg} / \mathrm{m}^{3} \quad \mathrm{~g}=9.81 \mathrm{~m} / \mathrm{s}^{2} \quad \mu=10^{-3} \mathrm{~kg} / \mathrm{ms}$

\section{Résultats}

Le modèle a été adapté au cas du clapage en modifiant l'expression de la vitesse numérique de chute sédimentaire par une formulation de type suspension selon :

$w_{s f}=K_{s f}\left(C^{v}\right)^{n}$
$\left\{\begin{array}{l}n=1 \\ K_{s f}=-\alpha_{s f} w_{s}\end{array} \quad\left\{\begin{array}{l}w_{s f}=-\alpha_{s f} C^{v} w_{s} \\ w_{s}=f\left(D_{50}\right)\end{array}\right.\right.$

$C^{v}(g / l)$ Concentration initiale du rejet, $\alpha_{s f}$ Coefficient numérique propre aux simulations de clapage $\alpha_{s f}=0.0225, D_{50}$ Diamètre moyen des grains de sédiment composant le mélange, $w_{s}$ Vitesse sédimentaire d'une particule sédimentaire moyenne du mélange, $f$ est une fonction dépendant uniquement de la nature du mélange clapé soit : $f\left(D_{50}\right)\left\{\begin{array}{l}\text { Rejets } 100 \% \text { sable } \Rightarrow \text { formulation de Van Rijn } \\ \text { Rejets sablo vaseux } \Rightarrow w_{s}=\varphi_{s, \text { sable }} w_{s, \text { sable }}+\left(1-\varphi_{s, \text { sable }}\right) w_{s, \text { vase }} \\ \text { Rejets } 100 \% \text { vase } \Rightarrow \text { formulation de Stokes }\end{array}\right.$ 
La Figure 2 et la Figure 3 ci dessus montrent l'adéquation des résultats numériques par rapport à l'expérimentation en ce qui concernent les positions $\mathrm{Y}(\mathrm{m})$ et rayons $\mathrm{R}(\mathrm{m})$ ou diamètres $\mathrm{D}(\mathrm{m})$ du rejet au cours du temps.
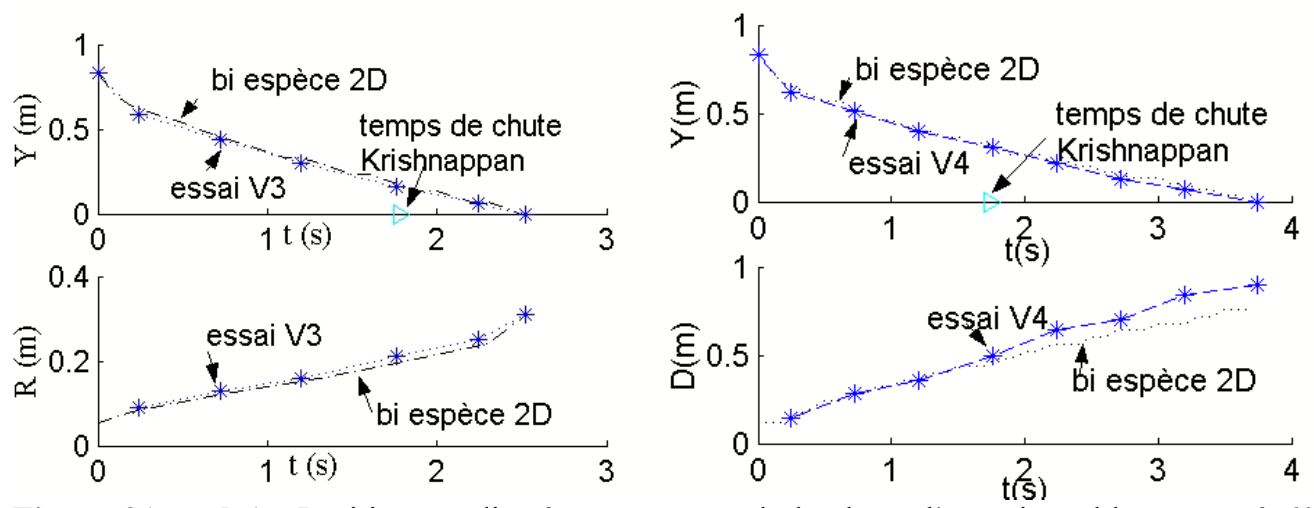

Figure 2(gauche). Positions et diamètres au cours de la chute d'un rejet sablo vaseux à $60 \%$ de sable de concentration volumique $350 \mathrm{~g} / \mathrm{l}$ sans courant ambiant.

Figure 3(droite). Positions et diamètres au cours de la chute d'un rejet sablo vaseux à $20 \%$ de sable de concentration volumique $350 \mathrm{~g} / \mathrm{l}$ dans un courant ambiant de $10 \mathrm{~cm} / \mathrm{s}$.

La Figure 2 présente le cas de 45 litres de rejet $60 \%$ sable ( $40 \%$ vase) de concentration volumique initiale $350 \mathrm{~g} / 1$, dans un canal au repos. La figure du haut montre les positions du rejet (position la plus basse) en fonction du temps au cours de la phase de chute pour l'essai expérimental concerné V3 et la simulation effectuée par notre modèle bi espèce 2D. La comparaison des données numériques et expérimentales montre la bonne représentation du modèle. La figure du bas donne le rayon du rejet (largeur maximum) en fonction du temps au cours de la chute; là encore l'adéquation des résultats est intéressante. On retrouve sur la Figure 3, le même type de représentation que sur la figure 2 avec 45 litres de rejet $20 \%$ sable $(80 \%$ vase) pour une concentration volumique initiale de $350 \mathrm{~g} / 1$ et un courant ambiant au sein du canal de $10 \mathrm{~cm} / \mathrm{s}$ et nous retrouvons encore une bonne adéquation des résultats numériques et expérimentaux.

Le Tableau 1 compare les temps de chute du modèle bi espèce 2D aux temps de chute de dix sept essais physiques différents. Dans la colonne de gauche, sont exprimées les caractéristiques $\mathrm{du}$ rejet expérimental: volume du rejet, concentration volumique initiale de sédiment, pourcentage de sable pour les rejets sablo vaseux, taille moyen des grains de sable et de vase, intensité du courant ambiant. Ces différents essais sont exprimés selon trois groupes permettant l'étude de l'influence du courant ambiant sur les rejets $100 \%$ sable, l'étude de l'influence de la concentration initiale $d u$ rejet et enfin l'importance $d u$ pourcentage de sable et du courant ambiant sur les rejets sablo vaseux. Sur les dix-sept simulations réalisées, le modèle bi espèce $2 \mathrm{D}$ donne une erreur absolue relative du temps de chute comprise entre 0 et 3 dixièmes de seconde. Sachant que l'incertitude du temps de chute expérimental peut atteindre 4 dixièmes de seconde le modèle bi espèce 2D fournit une approximation des temps de chute très satisfaisante : globalement l'erreur est inférieure à $10 \%$ pour un même jeu de calage. 


\begin{tabular}{|c|c|c|c|c|}
\hline $100 \%$ sable 60 litres $450 \mathrm{~g} / 1$ & $\mathrm{~T}(\mathrm{~s})$ chute & $\mathrm{T}(\mathrm{s})$ chute & Erreur & Erreur \\
\hline Influence du courant & essai & biesp2D & $\Delta \mathrm{t}(\mathrm{s})$ & $\%$ \\
\hline $\mathrm{D}_{50}=90 \mu \mathrm{m} \quad \mathrm{Ua}=0 \mathrm{~m} / \mathrm{s}$ & 0.95 & 1.00 & 0.05 & $5.26 \%$ \\
\hline $\mathrm{D}_{50}=160 \mu \mathrm{m} \quad \mathrm{Ua}=0 \mathrm{~m} / \mathrm{s}$ & 0.72 & 0.70 & 0.02 & $2.77 \%$ \\
\hline $\mathrm{D}_{50}=90 \mu \mathrm{m} \quad \mathrm{Ua}=0.1 \mathrm{~m} / \mathrm{s}$ & 1.54 & 1.60 & 0.06 & $3.89 \%$ \\
\hline $\mathrm{D}_{50}=90 \mu \mathrm{m} \quad \mathrm{Ua}=0.2 \mathrm{~m} / \mathrm{s}$ & 1.60 & 1.60 & 0.00 & $\mathbf{0 . 0 0 \%}$ \\
\hline $\mathrm{D}_{50}=90 \mu \mathrm{m} \quad \mathrm{Ua}=0.25 \mathrm{~m} / \mathrm{s}$ & 2.20 & 2.00 & 0.20 & $9.09 \%$ \\
\hline $100 \%$ sable 4,5 litres $\mathrm{Ua}=0 \mathrm{~m} / \mathrm{s}$ & $\mathrm{T}(\mathrm{s})$ chute & $\mathrm{T}(\mathrm{s})$ chute & Erreur & Erreur \\
\hline $\mathrm{D}_{50}=90 \mu \mathrm{m}$ Influence de $C$ & essai & biesp2D & $\Delta \mathrm{t}(\mathrm{s})$ & $\%$ \\
\hline $\mathrm{C}=100 \mathrm{~g} / 1$ & 3.40 & 3.50 & 0.10 & $2.94 \%$ \\
\hline $\mathrm{C}=100 \mathrm{~g} / 1$ & 3.80 & 3.50 & 0.30 & $7.89 \%$ \\
\hline $\mathrm{C}=100 \mathrm{~g} / \mathrm{l}$ & 3.70 & 3.50 & 0.20 & $5.40 \%$ \\
\hline $\mathrm{C}=400 \mathrm{~g} / \mathrm{l}$ & 1.60 & 1.70 & 0.10 & $6.25 \%$ \\
\hline$C=200 \mathrm{~g} / 1$ & 2.47 & 2.60 & 0.13 & $5.26 \%$ \\
\hline $\mathrm{C}=200 \mathrm{~g} / 1$ & 2.53 & 2.60 & 0.07 & $2.76 \%$ \\
\hline Sablo vaseux 45 litres $350 \mathrm{~g} / 1$ & $\mathrm{~T}(\mathrm{~s})$ chute & $\mathrm{T}(\mathrm{s})$ chute & Erreur & Erreur \\
\hline$D_{50}($ sable $)=90 \mu \mathrm{m}$ D $50($ vase $)=15 \mu \mathrm{m}$ & & & & \\
\hline Influence \% sable et courant & essai & biesp2D & $\Delta \mathrm{t}(\mathrm{s})$ & $\%$ \\
\hline $20 \%$ sable $\mathrm{Ua}=0 \mathrm{~m} / \mathrm{s}$ & 2.80 & 2.70 & 0.1 & $3.57 \%$ \\
\hline $60 \%$ sable $\mathrm{Ua}=0 \mathrm{~m} / \mathrm{s}$ & 2.52 & 2.50 & 0.02 & $0.79 \%$ \\
\hline $20 \%$ sable Ua $=0.1 \mathrm{~m} / \mathrm{s}$ & 3.74 & 3.70 & 0.04 & $1.07 \%$ \\
\hline $60 \%$ sable $\mathrm{Ua}=0.1 \mathrm{~m} / \mathrm{s}$ & 3.53 & 3.50 & 0.03 & $\mathbf{0 . 8 5 \%}$ \\
\hline $100 \%$ sable $\mathrm{Ua}=0 \mathrm{~m} / \mathrm{s}$ & 1.76 & 1.80 & 0.04 & $2.27 \%$ \\
\hline $100 \%$ sable Ua $=0.1 \mathrm{~m} / \mathrm{s}$ & 2.02 & 2.00 & 0.02 & $0.99 \%$ \\
\hline
\end{tabular}

Tableau 1. Comparaison des temps de chute de rejets $100 \%$ sable ou sablo vaseux avec ou sans courant ambiant.

En adéquation avec les observations expérimentales, nous retrouvons d'autre part dans les résultats du modèle, l'augmentation sensible du temps de chute avec l'intensité du courant ambiant et ce pour tous les types de rejets. De même, le modèle suit correctement l'influence de la concentration initiale avec une nette diminution du temps de chute au fil de l'augmentation de la concentration sédimentaire. Enfin, nous observons bien également une augmentation sensible du temps de chute avec la diminution du pourcentage de sable dans les rejets sablo vaseux.
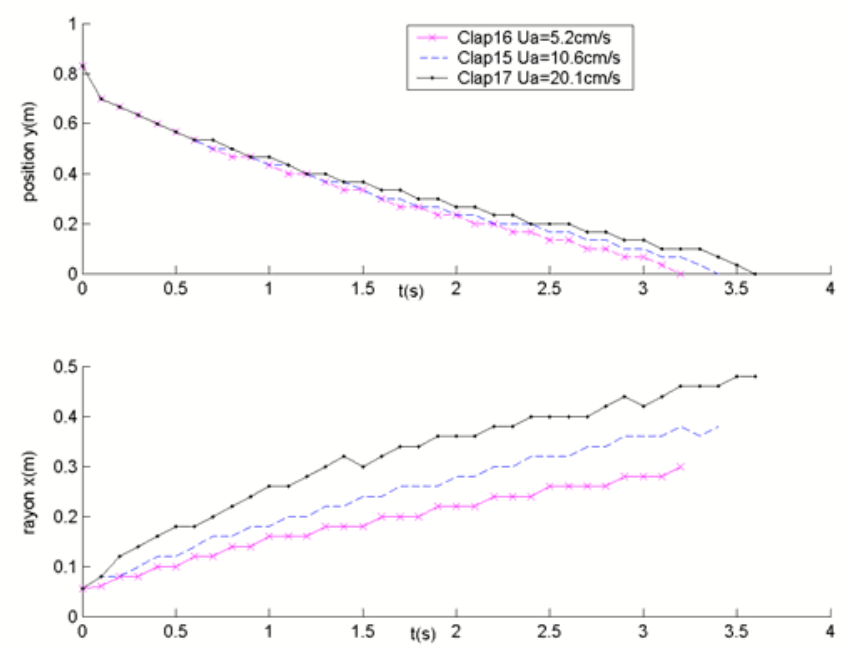

Figure 4 Influence du courant ambiant sur la phase de chute de rejet $100 \%$ vase. 
La Figure 4 propose la comparaison de trois simulations de 45 litres de rejets $100 \%$ vase de diamètre $10 \mu \mathrm{m}$ et de concentration volumique initiale fixée à 350g/l. Clap 15, Clap 16 et Clap 17 sont les noms donnés aux essais physiques $\left(\right.$ Farout-Fréson $^{7}$ ) correspondant à des courants ambiants respectifs de 10,6 cm/s, $5,2 \mathrm{~cm} / \mathrm{s}$ et $20,1 \mathrm{~cm} / \mathrm{s}$. Malgré le fait que le modèle bi espèce $2 \mathrm{D}$ ne tienne pas compte de la rhéologie spécifique des sédiments cohésifs, l'influence du courant ambiant sur la phase de chute est correctement reproduite et nous assistons bien à une augmentation du temps de chute et du rayon du rejet à l'impact avec l'augmentation du courant ambiant au sein du canal.

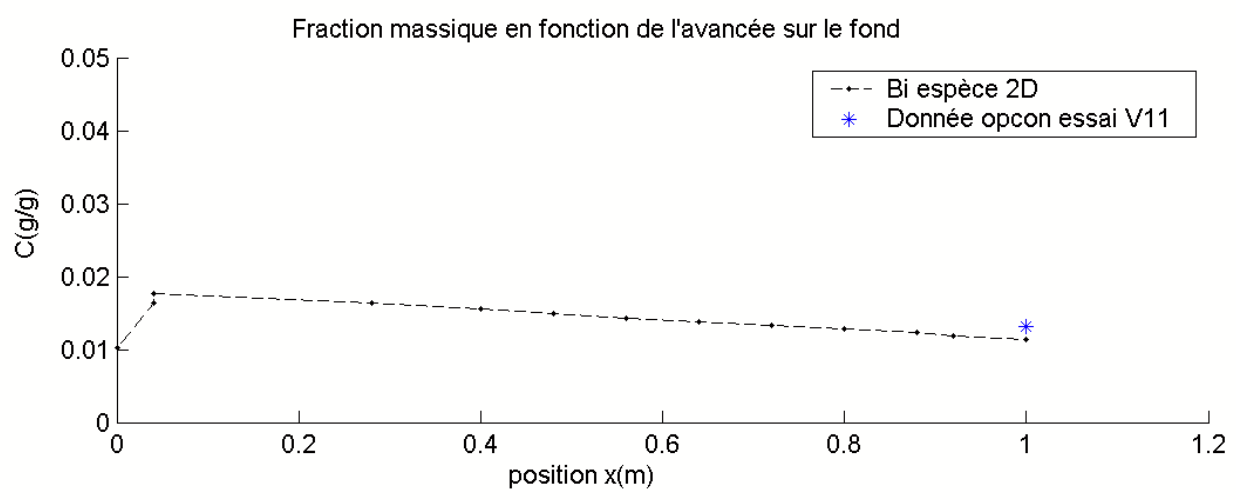

Figure 5 Phase de transport d'un rejet 100\% sable sans courant ambiant : fraction massique de sédiment transportée sur le fond.

Ce modèle permet aussi d'approcher les deux autres phases du clapage avec l'impact au sol et le transport des sédiments par courant de densité et les premiers résultats numériques obtenus (intensité du courant de densité et concentration de sédiment transportée sur le fond) sont encourageants : la Figure 5 donne la fraction massique maximum de sédiment transporté par courant de densité en fonction de l'avancée du rejet sur le fond pour un rejet $100 \%$ sable sans courant (la valeur OPCON expérimentale est de $13 \mathrm{~g} / 1$ à 1 mètre de l'axe du rejet contre une valeur de $11.40 \mathrm{~g} / \mathrm{l}$ pour le modèle bi espèce $2 \mathrm{D}$ ).

\section{Conclusion}

Les résultats du modèle sur la phase de chute de différents types de rejets de dragage par clapage montrent que les différentes positions du nuage obtenues par le modèle bi espèce $2 \mathrm{D}$ sont en quasi adéquation avec les données expérimentales. Les temps de chute numériques sont en accord avec les temps de chute expérimentaux dans la totalité des simulations effectuées et les influences du courant ambiant, de la concentration initiale ou du pourcentage de sable dans les rejets sablo vaseux sont respectées. La vitesse de chute numérique proposée, étant exclusivement définie en fonction des données initiales du rejet considéré, donne un caractère opérationnel au modèle bi espèce $2 \mathrm{D}$ de clapage. Cette nouvelle approche de la modélisation de la vitesse de chute sédimentaire permet ici une bonne reproduction du comportement du rejet qu'il soit $100 \%$ sable ou sablo vaseux dans un milieu avec ou sans courant ambiant. L'influence du courant 
ambiant est parfaitement reproduite pour les rejets de type 100\% vase malgré le fait que le modèle bi espèce $2 \mathrm{D}$ ne tienne pas compte de la rhéologie des sédiments cohésifs. Et dans son ensemble le modèle bi espèce $2 \mathrm{D}$ est cohérent pour toute la phase de chute de tous les types de clapage

Remerciements : L'auteur principal tient ici à remercier la Région Picardie pour le financement de ce travail de novembre 2000 à juin 2003.

\section{Bibliographie}

1 Anderson T.B., Jackson J. (1967). A fluid mechanical description of fluidized beds , Ind. Eng. Chemical Fundamental, vol. 6, n 4 p. 527-539.

2 Boutin R. (1999). Amélioration des connaissances sur le comportement des rejets en mer de produits de dragage de type vase, phénomènes à court terme et dans le champ proche. Thèse de doctorat de l'Institut National des Sciences Appliquées de Lyon 527 p.

3 Brandsma M.G., Divoky D.J. (1976). Development of models for prediction of Short Term FATE of dredged material discharged in the estuarine environment Tetra Tech Inc. for USAEWES Contract report D-76-5 $133 \mathrm{p}$

4 Burel D., Villaret C., (2000). Modélisation de la dispersion des rejets par clapage projet LITEAU rapport EDF HP-72/2000/054/A.

5 Dhatt G., Touzot G., Lefrançois E. (2005). Méthode des éléments finis Édition Hermès $595 \mathrm{p}$.

6 Drapeau G., Lavallee D., Dumais J.F., Walsh G. (1992). Dispersion model of dredge spoil dumped in coastal water » Coastal engineering Venice Italy 4-9 Oct. 1992 p. $3054-3067$

7 Farout Fréson I. (2004). Modélisation du clapage en mer; étude du champ proche; chute et transport sur le fond. Thèse de doctorat de l'Université de Technologie de Compiègne LHN $315 \mathrm{p}$.

8 Gordon R.B. (1974). Dispersion of dredged spoil dumped in near-shore waters Estuarine and coastal marine science tome 2 pp 349-358

9 Krishnappan B.G. (1976). Dispersion of granular material dumped in deep water Burlington, Ontario: environment Canada scientific series n ${ }^{\circ} 55,113 \mathrm{p}$.

10 Migniot C. (1989). Étude des propriétés physiques de différents sédiments très fins et de leur comportement sous des actions hydrodynamique La Houille Blanche, $\mathrm{n}^{\circ}$, pp 591-619

11 Richardson Y.F., Zaki W.N. (1954). Sedimentation and fluidization: part I Trans. Inst. Chem. Engineering Vol 32, pp 35-53

12 Tambo N., Watanabe Y. (1979). Physical characteristics of flocs I: The floc density function and aluminium floc Water Research Vol 13, pp 409-419

13 Van Leussen W. (1993). Field measurements of flocs sizes and settling velocities, MAST G8M, Workshop Leuven, 5-6 April 1993

14 Villaret C., Lekien M., Claude B., Vinet F. (1997). Étude expérimentale de la dispersion des rejets par clapage d'un mélange de sable et de vase (influence du courant et de la concentration). Rapport EDF HE-42/97/072/A 